\title{
Microwave-Assisted Rapid Extracellular Biosynthesis of Silver Nanoparticles Using Carom Seed (Trachyspermum copticum) Extract and in Vitro Studies
}

\author{
Deshpande Raghunandan ${ }^{1}$, Prashant Arunkumar Borgaonkar ${ }^{2}$, Basawaraj Bendegumble ${ }^{1}$, Mahesh \\ Dhondojirao Bedre ${ }^{3}$, Mantripragada Bhagawanraju ${ }^{4}$, Manjunath Sooganna Yalagatti ${ }^{5}$, Do Sung Huh ${ }^{6}$, \\ Venkataramana Abbaraju ${ }^{3}$ \\ ${ }^{1}$ H.K.E.S's College of Pharmacy, Gulbarga, India \\ ${ }^{2}$ R.M.E.S College of Pharmacy, Gulbarga, India \\ ${ }^{3}$ Materials Chemistry Laboratory, Department of Material Science, Gulbarga University, Gulbarga, India \\ ${ }^{4}$ CM College of Pharmacy, Hakimpet, Hyderabad, India \\ ${ }^{5}$ Institute of Pharmaceutical Science, Siddipeth, India. \\ ${ }^{6}$ Department of Biomedicinal Chemistry, Inje University, Kimhae, South Korea \\ E-mail: raman_chem@rediffmail.com \\ Received February 28, 2011; revised March 29, 2011; accepted April 15, 2011
}

\begin{abstract}
Microwave-assisted rapid extracellular biosynthesis of silver nanoparticles was carried out by using carom seed (Trachyspermum copticum) extract as the reducing agent. The reaction mixture containing $\mathrm{AgNO}_{3}$ and carom seed extract when exposed to microwave irradiation resulted in reducing silver ions to bio-functionalized silver nanoparticles of size 6- $50 \mathrm{~nm}$. The AgNP were characterized by UV-vis spectroscopy (UV-vis), X-ray diffraction (XRD), energy dispersive X-ray analysis (EDAX), field emission scanning electron microscopy (FESEM), transmission electron microscopy (TEM) and atomic force microscopy (AFM). Themogravimetric analysis (TGA) and fourier transform infrared spectroscopy (FTIR) are used to understand the possible mechanism of biosynthesis. In this study, we have also investigated the antimicrobial and antioxidant activities of bio-functionalized AgNP. The antibacterial activity is investigated by measuring the zone of inhibition and antioxidant study is done using 1,1-diphenyl-2-picryl hydrazyl (DPPH) method.
\end{abstract}

Keywords: Microwave, Silver Nanoparticles, Biosynthesis, Carom Seed (Trachyspermum copticum), Antibacterial Activity, Antioxidant Activity

\section{Introduction}

The perfection to unfold and view the structure and function of life at the nano level is leading to rapid development in technology [1] and health sciences [2]. With bottom-up synthesis approach, tailoring of metal particles at nanoscale is expected to open new protective and preventive aspects from life threatening diseases [3]. Metal nanoparticles, which have a high specific surface area, have been studied extensively because of their remarkable physicochemical characteristics like catalytic, optoectronic and magnetic properties [4]. It can be expected that the high fraction of surface atoms of silver nanoparticles (AgNP) will lead to an excellent antimicrobial activity when compared to bulk Ag metal [5]. This can be attrib- uted to the cohesion between the nanoparticles surface and the microbial cells, and hence is found to be size dependent. AgNP are currently used as active drug in targeted drug delivery [6], gene delivery [7] and artificial implants [8] and as a diagnostic agent in imaging and sensors for detection of different diseases in their early stages. Owing to their mutation-resistant antimicrobial activity, they are being used in different pharmaceutical formulations as antibacterial clothing [9], burn ointments [10] and as coating for medical devices [11].

Methods of nanoparticle production through different physical and chemical routes have their own demerits as they produce enormous environmental contaminations and hazardous byproducts. Thus, there is a need for "green chemistry" that ensures clean, non-toxic, and 
environment-friendly methods. The increasing demand for functionalized nanoparticles has encouraged developing new bio-routes. These include employing microorganisms, such as: Fusarium oxysporum [12], Fusarium semitactum [13], Cladosporium Sp. [14], and also different plants like alfalfa [15] neem [16] and clove [17].

In this paper, we report on the microwave-assisted rapid synthesis of highly stable bio-functionalized AgNP using carom seed extract as a reducing agent. Carom seed commonly cultivated in India and adjacent countries as the local environment suits for their growth. It is used for many domestic and medicinal purposes like diarrhea, dyspepsia, cholera, flatulence, and indigestion. Carom seeds are also extensively used in Ayurvedic medicine and Unani systems [18]. In literature we have not come across using this type of seed for formation of stable $\mathrm{AgNP}$ in aqueous system.

The formation of AgNP is understood from the UV-vis spectroscopy and X-ray diffraction studies. Transmission electron microscope studies indicate that AgNP are in the rage of $6-50 \mathrm{~nm}$ and most of them are nearly spherical in shape. Interestingly the colloidal suspension of AgNP is stable for $18-20$ weeks, much greater than the stability of nanoparticles synthesized from microorganisms [12-14]. Use of microwave exposed extracellular carom seed extract carries high level reproducibility.

In recent years, the antimicrobial resistance has emerged as a major public health problem. The metallic AgNP show lethal effect on the verity of microorganisms and do not allow the pathogens to develop resistance unlike conventional and narrow spectrum antibiotics. There lies a strong challenge to produce stable and safe AgNP to prevent bacterial growth significantly. Though the antibacterial activity of AgNP is being studied extensively, reports on the effect of these bio-functionalized nanoparticles in particular are rare. Free-radical involvement of AgNP surface in antimicrobial activity is discussed based on their zone of inhibition. AgNP synthesized by this process can be used as an effective tool in the control of microorganisms at a very low concentration and as a preventive agent in deleterious infections. The free-radical effect of AgNP is compared with the well known antioxidant, butyl hydroxy anisole (BHA) both at the same concentration which is determined using DPPH method. Our results support simple and cost-effective production of stable functionalized AgNP which are suitable for formulation of new types of bactericidal medicines.

\section{Materials and Methods.}

\subsection{Materials Used}

$\mathrm{AgNO}_{3}$ and analytical grade $\mathrm{C}_{2} \mathrm{H}_{5} \mathrm{OH}$ and butyl hydroxy anisole (BHA) is procured from Himedia Laboratories. Highly pure carom seeds are procured from Agricultural University, Dharwad. Double distilled water is used throughout the work. DPPH and the readymade agar media was procured from Sigma Chemicals, U.S.A.

\subsection{Silver Nanoparticles (AgNP) Biosynthesis Using Carom Seed}

Established procedure for the extraction of carom seed essential oils is adopted to prepare the aqueous ethanolic extracellular extract $\left(\mathrm{C}_{2} \mathrm{H}_{5} \mathrm{OH}: \mathrm{H}_{2} \mathrm{O}:: 1: 1\right)$ [19]. For preparing the aqueous extracellular solution, $1 \mathrm{~g}$ freshly collected and perfectly dried carom seed coarse powder was taken and added to a solution mixture containing $50 \mathrm{ml}$ of ethanol and $50 \mathrm{ml}$ double deionized water in a $250 \mathrm{ml}$ wide neck Borosil conical flask. The conical flask containing the reaction mixture was kept on a shaker for $4 \mathrm{~h}$. Then the aqueous extracellular filtrate of carom seeds was obtained by passing it through Whatman filter paper no. 40 . The clear filtrate contains only soluble organic moieties of the seed and the solid residue is discarded. This aqueous ethanolic filtrate is the extracellular extract of carom seed used for the reduction process. Exactly $5 \mathrm{ml}$ of the resultant clear extract was added to $50 \mathrm{ml}$ carefully weighed $10^{-3} \mathrm{M} \mathrm{AgNO}_{3}$ aqueous solution in a $250 \mathrm{ml}$ Borosil flask and exposed to microwave unifrequency radiation (DAEWOO, $2.45 \mathrm{GHz}$ ) for the reduction process to take place.

\subsection{Analysis}

Periodically, upper layer of the reaction mixture is taken for UV-vis spectroscopy observation which was performed on an ECIL 5704SS UV-visible spectrophotometer at a resolution of $1 \mathrm{~nm}$. For crystallinity studies, $\mathrm{X}$-ray diffraction (XRD) measurement of the biosynthesized solution with a drop coated on glass substrate bio-film was carried out on a Siemens X-ray diffractometer (Japan) instrument operated at $30 \mathrm{kV}$ and a current of $20 \mathrm{~mA}$ with $\mathrm{Cu} K \alpha .(1=1.54 \AA)$ radiation. The morphology of the AgNP was examined using field emission scanning electron microscopy (FESEM, FEI Nova nano 600 , Netherlands), and for this, the images were operated at $15 \mathrm{kV}$ on a $0^{\circ}$ tilt position. Transmission electron microscopy (TEM) image of the sample was obtained using Technai-20 Philips transmission electron microscope operated at $190 \mathrm{keV}$. Atomic force microscopy (AFM) images were collected under ambient conditions on a Veeco Innova scanning probe microscope. Etched $\mathrm{Si}$ nanoprobe tips (RTESPA-M) were used for the same. For fourier transformed infrared radiation (FTIR) spectroscopy measurements AgNP powder sample was pre- 
pared by centrifuging the synthesized AgNP solution at $10,000 \mathrm{rpm}$ for $15 \mathrm{~min}$. The solid residue layer which contains AgNP was redispersed and washed in sterile deionized water for three times to remove the unattached biological impurities. The pure residue was then dried perfectly in an oven overnight at $65^{\circ} \mathrm{C}$. Thus obtained powder was subjected to FTIR measurements carried out on a Perkin-Elmer Spectrum-One instrument at a resolution of $4 \mathrm{~cm}^{-1}$ in $\mathrm{KBr}$ pellets.

\subsection{In Vitro Activity}

\subsubsection{Antibacterial Activity.}

The culture media is prepared with peptone- $10 \mathrm{~g}, \mathrm{NaCl}$ $10 \mathrm{~g}$ and yeast extract $5 \mathrm{~g}$, agar $20 \mathrm{~g}$ in $1000 \mathrm{ml}$ of distilled water and boiled. Initially, the stock cultures of Bacillus subtilis, methicillin resistant Staphylococcus aureus (MRSA), Pseudomonas aerosenosa and Salmonella typh $i$ were revived by inoculating in broth media in separate test tube and grown at $37^{\circ} \mathrm{C}$ for $18 \mathrm{~h}$. These microorganisms were selected on the basis of their patho genicity, resistance and severity in forming infections. The required volume of test sample $(2.5,5,10,20 \mu \mathrm{g} / \mathrm{mL})$ was added and mixed well. The media was poured into the pre-autoclaved petri dishes. The $104 \mathrm{CFU}$ culture was inoculated and grown at $37^{\circ} \mathrm{C}$ for $24 \mathrm{~h}$. The control plate is prepared with respective bacteria without AgNP samples for comparative studies.

\subsubsection{Free-Radical Scavenging Activity.}

Both the functionalized AgNP solution and butylated hydroxy anisole (BHA) $(2.5 \mu \mathrm{g}, 5 \mu \mathrm{g}, 7.5 \mu \mathrm{g}$ and $10 \mu \mathrm{g})$ were taken in different test tubes. The volume was adjusted to $1000 \mu \mathrm{l}$ by adding methanol. Five milliliters of a $0.1 \mathrm{mM}$ methanolic solution of 1,1-diphenyl-2-picryl hydrazyl (DPPH) was added to these tubes and shaken vigorously. The tubes were allowed to stand at $27^{\circ} \mathrm{C}$ for $20 \mathrm{~min}$. The control was prepared as above without addition of AgNP aqueous solution. The absorbance of the functionalized AgNP was measured at $517 \mathrm{~nm}$ with UV-vis spectroscopy. Free-radical scavenging activity was calculated using the following formula:

$$
\% \text { radical scavenging activity }=\left[\frac{(\text { control } O D-\text { functionalized silver solution } O D)}{\text { control }} O D\right] \times 100
$$

\section{Results and Discussion}

\subsection{Bio-reduction and Characterization.}

The detailed study on microwave-assisted extracellular biosynthesis of AgNP using ethanolic aqueous carom seed (T. copticum) extract was carried out with the antibacterial and antioxidant effects. The initial color of the solution after the addition of carom seed extract to the aqueous $\mathrm{AgNO}_{3}$ solution was nearly colorless. In the first phase, the intensity of the reaction mixture on exposure to microwave radiation increases exponentially with time. The metal ions reduction occurs very rapidly and more than $90 \%$ of the reduction of $\mathrm{Ag}^{+}$ions will be completed in 90 seconds. From 90 to $150 \mathrm{sec}$ the reaction phase is drastically reduced and the reaction rate changes to linear phase. After $150 \mathrm{sec}$, the reaction stops as the intensity of the reaction shows almost a parallel line with $\mathrm{x}$-axis with respect to time. The change in color of the reaction mixture is noted at every $10 \mathrm{sec}$ interval and is shown in the inset of Figure 1. Colorlessness of reaction mixture at the initial stage and the final deep reddish-brown color after the completion of the reaction are also shown. The absorbance intensity, that is, the formation of AgNP will increase with increased exposure of reaction mixture to microwave and is shown in Figure 1(a). The microwave exposed methodology is much faster than the earlier conventional studies using other plants extracts [12-14] and microorganisms [15-17]. The time required for the conventional synthesis of AgNP from other plants was 2 $-4 \mathrm{~h}$ and from bacteria was $24-120 \mathrm{~h}$ and are thus rather slow.

It is well known fact that microwave produces super heating non-ionizing radiations at ambient pressure [20]. The accelerated rate of reaction is also attributed to the strong agitation and reorientation of the dipolar water molecules, electron rich biological moieties and conducting silver ions which tremendously enhance the possibility of collision between them.

Figure 1(b) shows the UV-vis spectrum of the reaction mixture. The peak at $255 \mathrm{~nm}$ is attributed to the absorption band for the water soluble organic moieties present in the extract. This also indicates that the organic moieties are involved in the reduction process of ions to nanoparticles. The color developed in the solution, as a result of AgNP formation was observed with a surface plasmon resonance (SPR) peak in the UV-vis spectroscopy. Reddish brown color of the AgNP arises due to SPR vibrations in the metal nanoparticles. It is seen from the spectra that the SPR of AgNP band occurs at $465 \mathrm{~nm}$ [21]. The hyperchromic shift of this peak with increased exposure to microwave is found to be directly depending 


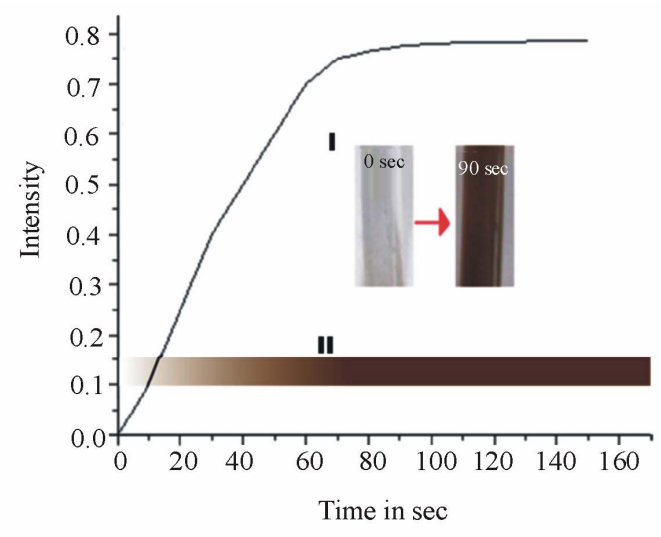

(a)

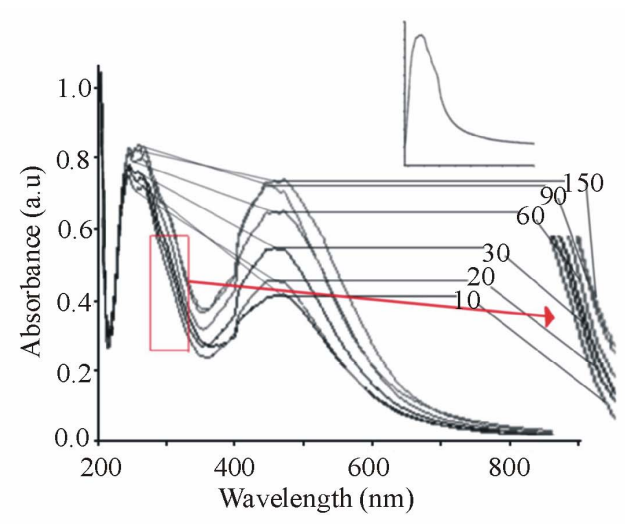

(b)

Figure 1. (a) Graph showing change in color intensity of the reaction mixture with respect to time. Inset: I. Initial and the final color of the reaction mixture containing aqueous $10^{-3} \mathrm{M} \mathrm{AgNO}_{3}$ solution and aqueous ethanolic carom seed extract on microwave irradiation. II. Change in the color of the reaction mixture with time in sec. (b) UV-visible spectra of AgNP biosynthesis, absorbance recorded as a function of time. Projected and enlarged view of UV spectra is highlighted with an arrow line to show the stoppage of reaction at 90 sec. Inset: UV-vis spectrum of the extracellular carom seed extract.

upon the concentration of AgNP formed. In addition to $465 \mathrm{~nm}$ peak, another peak at $480 \mathrm{~nm}$ also appears as a shoulder in the visible region after $60 \mathrm{sec}$ of the reaction. The $465 \mathrm{~nm}$ peak corresponds to the transverse plasmon vibration, whereas, the peak at $480 \mathrm{~nm}$ is due to excitation of longitudinal plasmon vibrations. Wavelengths of these peaks are different, distinctly separated which indicate that AgNP formed in the solution have different sizes and shapes and are in aggregates form [22]. The broadening and splitting of the SPR with the increase in microwave exposure is probably due to the dampening of surface plasmon caused by a resonance change [23] which in turn is due to the change in the refractive index of the surrounding medium and increase in the size of AgNP in the colloidal solution. An absorption band at $255 \mathrm{~nm}$ is clearly visible and is attributed to electronic excitation of organic moieties. In order to verify the results of the UV-vis analysis, the sample of bio-reduced AgNP was examined by XRD, which gave sharp, crystalline peaks of $\mathrm{Ag}$ as shown in Figure 2. The different facet markings will agree with the standard JCPDS report. The appearance of "Al" in figure is because of the aluminum grid base used for the analysis. The diffraction pattern also suggests that the AgNP formed are polycrystalline in nature. The study of metallic nature of these AgNP is further strengthened by EDAX image shown in the inset of Figure 2.

Figure 3 shows FESEM images of functionalized AgNP. It can be seen that they are thickly coated with organic moieties on them with core shell morphology of size $6-50 \mathrm{~nm}$. In Figure 3(a), AgNP seem to be arranged in an organic matrix making it aqueous suspension. Higher resolution image at $300 \mathrm{~nm}$ (Figure 1) shows a group of particles in embedded in a organic moieties making a stable suspension. The particles appear to be polydispersed in nature and are roughly spherical in shape. Particles size distribution determined from the FESEM image, shown in the center as an inset of Figure 3 represents the histogram of the synthesized AgNP. It is observed that there is a marginal variation in the particle size. Almost $90 \%$ of the particles are in the range of $6-50 \mathrm{~nm}, 4 \%$ are in $51-60 \mathrm{~nm}$ and approximately $6 \%$ are in the $1-5 \mathrm{~nm}$ range. The preliminary studies indicate an encouraging fact that by making variation in the experimental parameters like $\mathrm{pH}$, concentration of the carom seed extract, frequency opted for microwave irradiation, and molar concentration $\mathrm{AgNO}_{3}$ will achieve the monodispersivity and uniformity in shape.

The clear morphology is reconfirmed with drop coated TEM grids and AFM images shown in Figure 4. Figure 4(a) shows a typical bright-field TEM image of the biosynthesized AgNP. The AgNP are nearly spherical in shape, and are in the range of $6-50 \mathrm{~nm}$ size indicating the dispersivity to be in a narrow range. On a careful observation we can see a sensitive layer adsorbed on the surface and between thin gaps of two nanoparticles (shown with arrow mark). We can infer that these are the organic moieties adsorbed on the surface and are responsible for inter particle binding. The same may also be responsible for bio-reduction of ions and formation of nanoparticles. Figure 4(b) shows a typical AFM representative image exhibiting the morphology of colloidal AgNP. Uniformity in the morphology of these nanoparticles may be attributed to the soft adsorbed layer and to the thick cover of organic moieties on the particles. AgNP appear to be higher in size $(100-120 \mathrm{~nm})$ than that are seen in TEM image. The most probable reason 


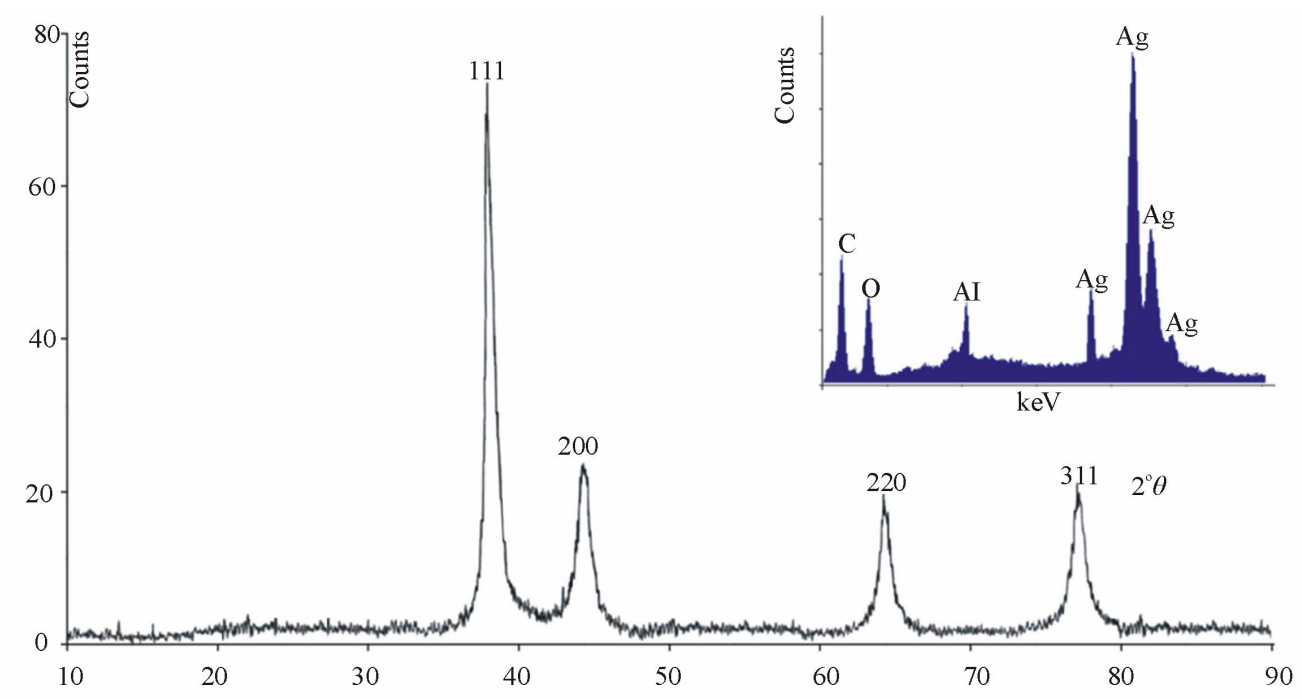

Figure 2. XRD pattern of crystalline AgNP synthesized using extracellular aqueous ethanolic carom seed extract. Inset fig. shows Energy dispersive x-ray spectrum EDAX of metallic biosynthesized AgNP.

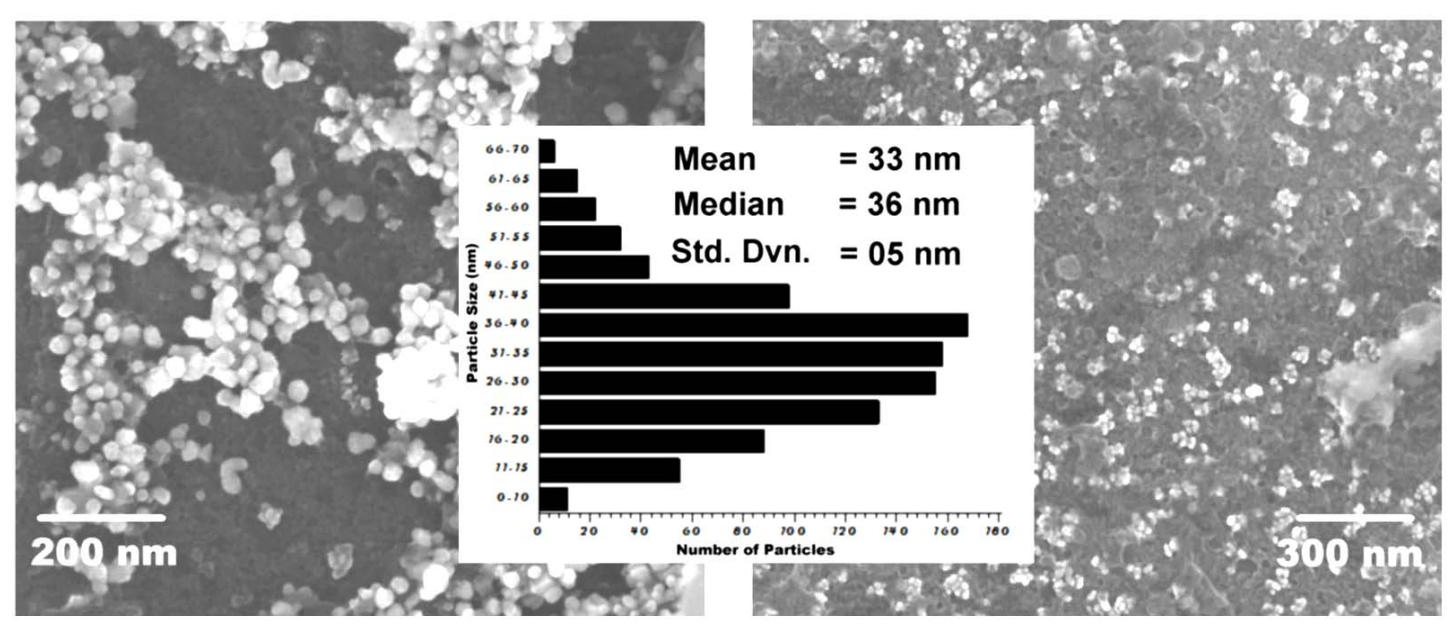

(a)

(b)

Fiure 3. (a)\& (b). FESEM images of synthesized AgNP in colloidal condition on different nanometric scale. Inset at the centre shows histogram indicating size distribution of AgNP.

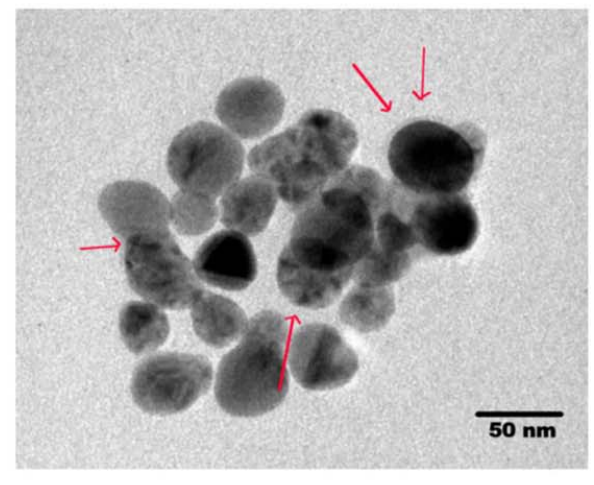

(a)

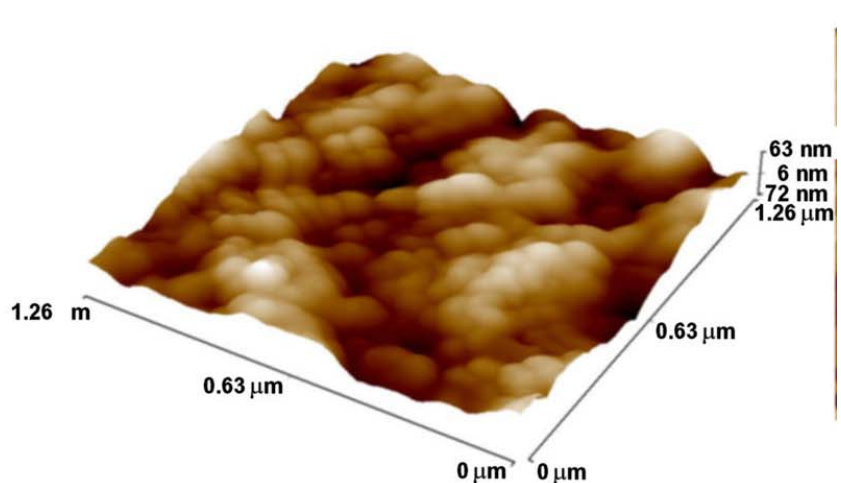

(b)

Figure 4. (a) TEM image of biosynthesized AgNP showing they are roughly spherical in shape. (b) Medium scale tapping mode AFM image of bio-functionalized AgNP adsorbed with organic layer. 
for increased size appearance may be due to the bio-adsorbed layer on the nanoparticles. This magnification can also be attributed to the convolution of the true particle size with AFM tip. The AFM data also show that the size of the particles depends on the deposition conditions.

Figure 5 is the TGA graph, shows three stages of weight loss. In I stage, from $0^{\circ} \mathrm{C}-100^{\circ} \mathrm{C}$, the weight loss of $4 \%$, is due to the evaporation of adsorbed water molecules and free $-\mathrm{OH}$ groups on the surface of the functionalized AgNP. Second weight loss of $18 \%$ from $100^{\circ} \mathrm{C}$ $-450^{\circ} \mathrm{C}$ is slow and steady and is attributed to the evaporation of absorbed water molecules. Third weight loss of $12.5 \%$ from $450^{\circ} \mathrm{C}-1100^{\circ} \mathrm{C}$ is due to the loss of strongly bound organic moieties layer present on the AgNP surface. The total weight loss of $34.5 \%$ from stage I-III gives confirmative evidence that the metallic core is thickly covered by bio-moieties shell. The undecomposed residue of $65.5 \%$ contains pure silver microstructures. The inset image shows the picture of pure silver microstructures after cooling the residue to ambient conditions. On further heating up to $1300^{\circ} \mathrm{C}$ the metal nanoparticles get melted to a liquid state. After cooling, pure spherical silver particles are formed due to the cohesive force in the molecules of the metal. These silver spheres are the bulk microstructures which possess the original color of the bulk silver.

Figure 6 shows FTIR spectrum of AgNP. FTIR shows peaks at 1741, 1641, 1569, 1460, 1263, 1099, 1020, $800 \mathrm{~cm}^{-1}$. This indicates secretion of some soluble organic components of carom seeds which could have contributed for the important role in the reduction and functionalization of the metal nanoparticles. Consequently the organic moieties adsorbed on the nanoparticles con-

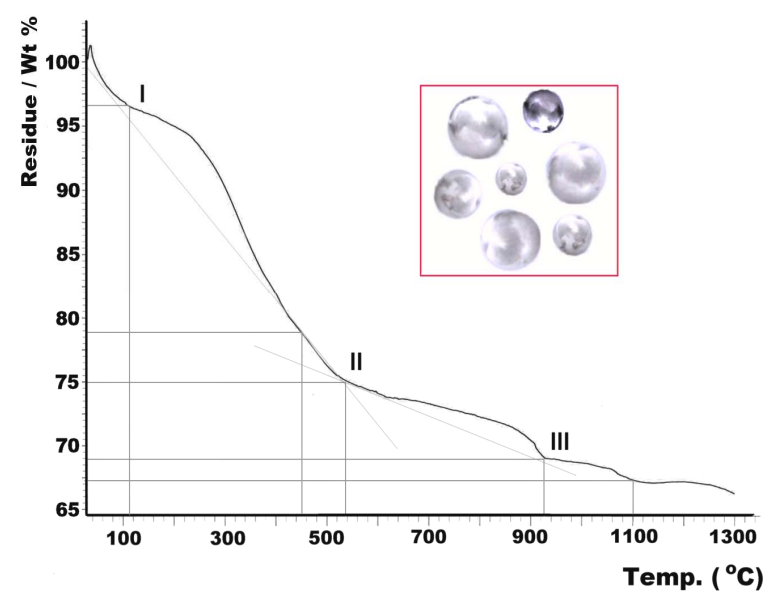

Figure 5. TGA graph showing the weight loss pattern of functional groups and other biological moieties adsorbed on the AgNP surface. Inset image shows bulk microstructures of pure silver as the residue left after cooling. fer the stability. We presume that the polyphenols like terpenoids (thymol, which is a major constituent of the essential oils) of carom seed show characteristic absorption peaks and the same are responsible for bio-reduction and capping process [24]. Peak at $1460 \mathrm{~cm}^{-1}$ is due to $\mathrm{C}-\mathrm{H}$ deformation of gem-dimethyl groups and 1099 and $1020 \mathrm{~cm}^{-1}$ are of $\mathrm{CH}_{3}-\mathrm{C}-\mathrm{CH}_{3}$ skeletal vibrations. $\mathrm{C}=\mathrm{C}$ stretching vibrations at $1641 \mathrm{~cm}^{-1}$ peak are due to aromatic rings. Conjugated $\mathrm{C}=\mathrm{C}$ bonds at $1569 \mathrm{~cm}^{-1}$ and bending vibration peak at $800 \mathrm{~cm}^{-1}$ suggest the presence of thymol adsorbed on the surface of AgNP. Among the major chemical constituents of carom seed (Thymol, P-Cymene and $\gamma$-Terpinene) [25], thymol is the only constituent which possesses aromatic ring in its structure. It appears that the same moiety could be adsorbed on the metal nanoparticle surface by with $\pi$-electrons. The peaks of phenolic $-\mathrm{OH}$ are seen at $3743 \mathrm{~cm}^{-1}$ and $-\mathrm{CH}_{3}$, $-\mathrm{CH}_{2}$, - $\mathrm{CH}$ stretching vibrations are observed at 2923, 2854 and $2690 \mathrm{~cm}^{-1}$ respectively.

\subsection{Antibacterial Activity and Free Radical Scavenging Activity}

Two different pathogens were chosen from each group of gram positive and gram negative segments for our studies. Pseudomonas aeruginosa can cause chronic opportunistic nosocomial infections which can't be treated with regular antibiotics. $P$. mirabilis causes maximum 'Proteus' infections. Methicillin-resistant Staphylococcus aureus (MRSA) causes infections which are difficult-to-treat. Typhoid is one of the serious infections developed from the simple strains of S. Typhi and is responsible for enteric fever.

AgNP synthesized with this green-clean technology, is exposed to all the strains on MHA plates treated with different concentrations (from $2.5 \mu \mathrm{g}$ to $20 \mu \mathrm{g} / \mathrm{mL}$ ) for studying antibacterial application as shown in Figure 7(a). The inhibitory effects of the sample are compared with the control plate prepared without addition of any drug. AgNP showed satisfactory growth inhibition effect against salmonella typhi, and significant growth inhibition is observed in all other pathogens. The zone of inhibition of AgNP in different microorganisms is different and is concentration-dependent. Inhibition in S. Typhi, $P$. aeruginosa, and B. subtilis is 5, 6 and $7 \mathrm{~mm}$ respectively at the highest concentration of $20 \mu \mathrm{g} / \mathrm{mL}$. MRSA is inhibited at the same concentration of AgNP but the rate of inhibition appears to be slow with increasing concentration compared to $B$. subtilis and $P$. aeruginosa. It is observed that the MIC of B. subtilis is higher than the other pathogens. The mechanism by which the nanoparticles are able to inhibit bacterial growth is not well understood, but it can be conceived that the AgNP affect the mem- 


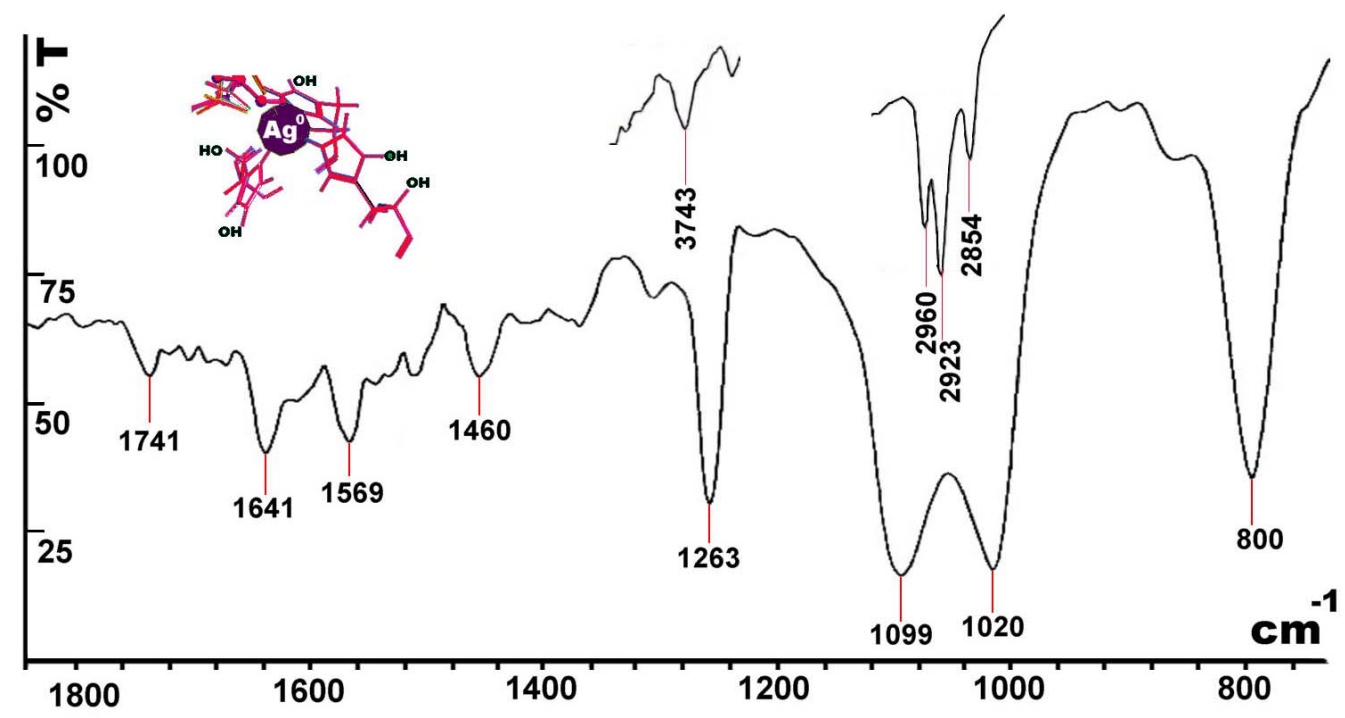

Figure 6. Typical FTIR absorption spectra of the bio-moieties of the macerated extracellular aqueous ethanolic carom seed extract adsorbed on the AgNP. Functionalized AgNP is shown as an inset.

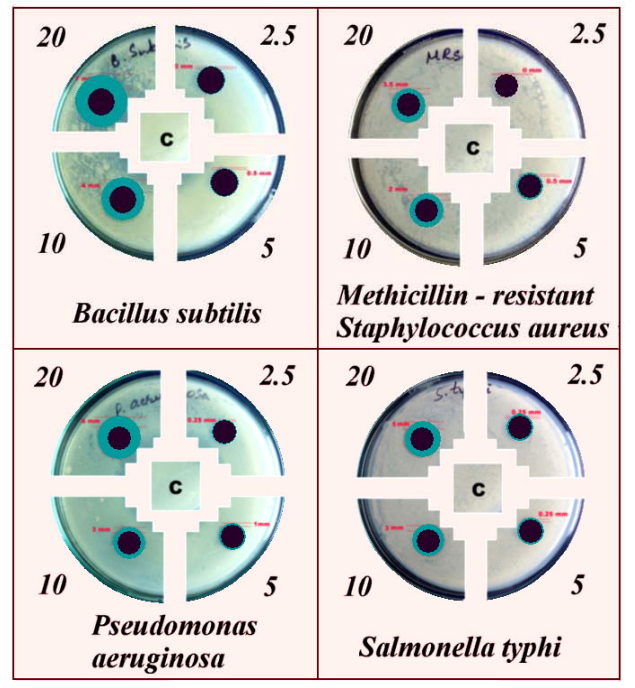

(a)

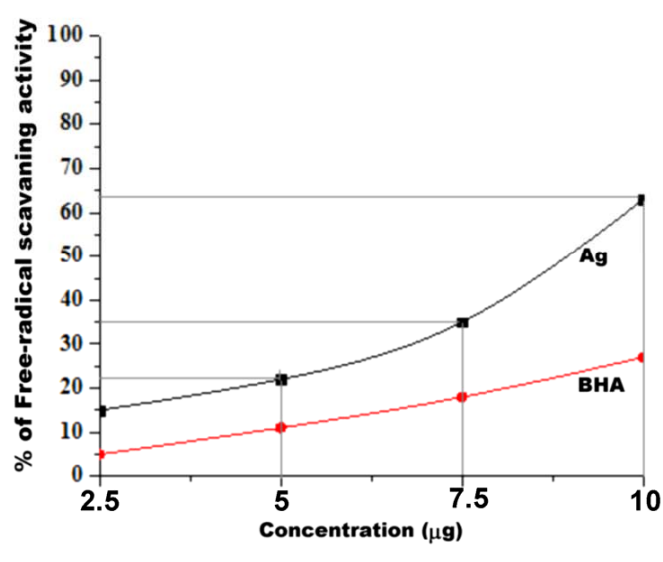

(b)

Figure 7. A. Antimicrobial activities of bio-functionalized AgNP using carom seed extract. For brevity only $1 / 4^{\text {th }}$ portion of the plates are shown. The center potion marked as " $C$ " shows control of respective microorganisms. All the concentrations are taken in $\mu \mathrm{g} / \mathrm{mL}$. B. Free-radical scavenging activity graph of AgNP and BHA indicating the quenching effect on DPPH radical at different $\mu$ g concentration.

brane of both the bacterial strains. It may lead to significant increase in the permeability and affect membrane transport. Also, there is no antimicrobial activity in the solution devoid of AgNP (produced out of carom seed extract) in the control plates shown in the center marked in figure as "C". Separate test is carried out for the antimicrobial analysis for carom seed extract only; even at the highest concentration, the effect is similar as seen in case of control plates. This study concludes that the antimicrobial effect is only due to synthesized AgNP. The study also infers that the functionalized AgNP using carom seed extract have good bactericidal activity at low concentration in different microbes. Further studies on the formulation of these nanoparticles, stability studies and other analysis for comparing the efficacy against commercial products are on the way.

Figure 7(b) shows very encouraging results. These functionalized AgNP have DPPH' free radical scavenging in a concentration dependent manner [26]. It could be seen from figure that at the same concentration AgNP 
scavenged the DPPH free radical five times more effectively than BHA. Even at concentration as low as $5 \mu \mathrm{g} / \mathrm{mL}$, where BHA has less than $10 \%$ efficiency, functionalized AgNP mopped up more than $40 \%$ free radicals in-vitro. Similarly the percentage of quenching effect on DPPH free radical was 9\% with AgNP at a minimum concentration of $2.5 \mu \mathrm{g}$, where BHA shows only $3 \%$ at same concentration. AgNP and BHA scavenged $60 \%$ and $12 \%$ respectively at a maximum concentration of $10 \mu \mathrm{g}$.

\section{Conclusions}

Carom seeds and silver are generally used as bactericidal agents; combination of both in the form of functionalized silver nanoparticles is envisaged. With the help of microwave-assisted top-down green chemistry approach, functionalized silver nanoparticles were synthesized in the range of $6-50 \mathrm{~nm}$ using aqueous ethanolic carom seed (Trachyspermum copticum) extract. Usage of AgNP thus produced are tested for in vitro applications and found to be more effective as protective and preventive antibacterial and antioxidant agent. Silver nanoparticles based on these findings may lead to valuable discoveries in various fields such as medicine and pharmaceutical research. As this method of biosynthesis is simple and handy; can be thought for commercial level of production.

\section{Acknowledgements}

Financial supports from BRNS (Grant No. 2009/34/ 14/BRNS), DST (Grant No.SR/S1/PC-10/2005) and UGC Major Research Project (33-307/2007 (SR) are acknowledged. We also acknowledge the help from Biogenics, Hubli for antimicrobial studies. We thank Prof. B. G. Mulimani, Vice-Chancellor, BLDE University, Bijapur for encouragement in the work. Raghunandan Deshpande thanks his father Shri. J. M. Deshpande for editing work \& Dr. Appala Raju, Principal of HKES college of pharmacy, Gulbarga for encouraging the research program.

\section{References}

[1] H. Devalapally, A. Chakilam and M. M. Amiji, "Role of Nanotechnology in Pharmaceutical Product Development," Journal of Pharmaceutical Sciences, Vol. 96, No. 10,2007 , pp. 2547-2565. doi.org/10.1002/jps.20875

[2] B. Semete, L. Booysen, Y. Lemmer, L. Kalombo, L. Katata, J. Verschoor and H. S. Swai, "In Vivo Evaluation of the Biodistribution and Safety of PLGA Nanoparticles as Drug Delivery Systems," Nanomedicine: Nanotechnology, Biology, and Medicine, Vol. 6, No. 1, February 2010, pp.
662-671. doi.org/10.1016/j.nano.2010.02.002

[3] P. K. Jain, X. Huang, H. Ivan, I. El-Sayed and M. A. El-Sayed, "Noble Metals on the Nanoscale: Optical and Properties and Some Applications in Imaging, Sensing, Biology, and Medicine," Accounts of Chemical Research, Vol. 41, No. 12, May 2008, pp. 1578-1586.

doi.org/10.1021/ar7002804

[4] H. Lu, E. L. Salabas and F. Schüth, "Magnetic Nanoparticles: Synthesis, Protection, Functionalization, and Application," Angewandte Chemie, International Edition, Vol. 46, No. 8, February 2007, pp. 1222-1244.

doi.org/10.1002/anie.200602866

[5] Y. Liu and L. Lin. "New Pathway for the Synthesis of Ultrafine Silver Nanoparticles from Bulk Silver Substrates in Aqueous Solutions by Sonoelectrochemical Methods," Electrochemistry Communications, Vol. 6, No. 11, November 2004, pp. 1163-1168.

[6] G. Aliev, J. Daza, A. Lipsitt, M. Martínez-Agüero, H. H. Palacios, K. Fischbach, M. E. Obrenovich, J. C. LaManna, V. Bragin and L. Morales, "Silver Nanoparticles as Alternate Strategies for Drug Delivery to Alzheimer Brain," Alzheimer's and Dementia, Vol. 5, No. 4, June 2009, p. 324.

[7] K. Roy, H. Q. Mao, S. K. Huang and W. Leong. "Oral Gene Delivery with Chitosan-DNA Nanoparticles Generates Immunologic Protection in a Murine Model of Peanut Allergy," Nature Medicine, Vol. 5, No. 4, 1999, pp. 387-391. doi.org/10.1038/7385

[8] E. Sachlos, D. Gotora and J. T. Czernuszka, "Collagen Scaffolds Reinforced with Biomimetic Composite Nano-Sized Carbonate-Substituted Hydroxyapatite Crystals and Shaped by Rapid Prototyping to Contain Internal Microchannels," Tissue Engineering, Vol. 12, No. 9, September 2006, pp. 2479-2487.

doi.org/10.1089/ten.2006.12.2479

[9] J. S. Kim, E. Kuk, K. N. Yu, J. Kim, S. J. Park, H. J. Lee, S. H. Kim, Y. K. Park, Y. H. Park, C. Hwang, Y. Kim, Y. Lee, D. H. Jeong and M. Cho, "Antimicrobial Effects of Silver Nanoparticles," Nanomedicine: Nanotechnology, Biology and Medicine, Vol. 3, No. 1, March 2007, pp. 95-101. doi.org/10.1016/j.nano.2006.12.001

[10] J. L. Elechiguerra, J. L. Burt, J. R. Morones, A. Camacho-Bragado, X. Gao, H. H. Lara and M. J. Yacaman, "Interaction of Silver Nanoparticles with HIV-1," Journal of Nanobiotechnology, Vol. 3, No. 6, June 2005, p. 6. doi.org/10.1186/1477-3155-3-6

[11] F. Furno, K. S. Morley, B. Wong, B. L. Sharp, P. L. Arnold, S. M. Howdle, R. Bayston, P. D. Brown, P. D. Winship and H. J. Reid, "Silver Nanoparticles and Polymeric Medical Devices: A New Approach to Prevention of Infection," Journal of Antimicrobial Chemotherapy, Vol. 54, No. 6, September 2004, pp. 1019-1924. doi.org/10.1093/jac/dkh478

[12] P. Mukherjee, S. Senapati, D. Mandal, et al., "Extracellular Biosynthesis of Silver Nanoparticles Using the Fungus Fusarium Oxysporum," Colloids and Surfaces B: Biointerfaces, Vol. 28, No. 4, May 2003, pp. 313-318. doi.org/10.1016/S0927-7765(02)00174-1 
[13] S. Basavaraja, D. S. Balaji, A. Lagashetty, A. H. Rajasab and A. Venkataraman, "Extracellular Biosynthesis of Silver Nanoparticles Using the Fungus Fusarium Semitectum," Materials Research Bulletin, Vol. 43, No. 5, 2008, pp. 1164-1170.

doi.org/10.1016/j.materresbull.2007.06.020

[14] D. S. Balaji, S. Basavaraja, D. Raghunandan, B. Mahesh, B. K. Prabhakar and A. Venkataraman. "Extracellular Biosynthesis of Functionalized Silver Nanoparticles by Strains of Cladosporium Cladosporioides Fungus," Colloids and Surfaces B: Biointerfaces, Vol. 68, No. 1, January 2009, pp. 88-92.

[15] L. J. Gardea-Torresdey, E. Gomez, R. J. Peralta-Videa, J. G. Parsons, H. Troiani and M. Jose-Yacaman, "Alfalfa Sprouts: A Natural Source for the Synthesis of Silver Nanoparticles," Langmuir, Vol. 19, No. 4, 2003, pp 13571361. doi.org/10.1021/la020835i

[16] S. S. Shankar, A. Rai, A. Ahmad and M. Sastry. "Rapid Synthesis of $\mathrm{Au}, \mathrm{Ag}$, and Bimetallic Au Core-Ag Shell Nanoparticles Using Neem (Azadirachta Indica) Leaf Broth," Journal of Colloid and Interface Science, Vol. 275, No. 2, May 2004, pp. 496-502. doi.org/10.1016/j.jcis.2004.03.003

[17] D. Raghunandan, S. Basavaraja, B. Mahesh, S. Balaji, S. Y. Manjunath and A. Venkataraman, "Rapid Biosynthesis of Irregular Shaped Gold Nanoparticles from Macerated Aqueous Extracellular Dried Clove Buds (Syzygium Aromaticum) Solution," Colloids and Surfaces B: Biointerfaces, Vol. 79, No. 1, August 2010, pp. 235-240. doi.org/10.1016/j.colsurfb.2010.04.003

[18] C. Thangham and R. Dhananjayan, "Antiinflammatory Potential of the Seeds of Carum Copticum Linn," Indian Journal of Pharmacology, Vol. 35, No. 6, 2003, pp. 388391.

[19] P. S. Murthy, B. B. Borse, H. Khanum and P. Srinivas, "Inhibitory Effects of Ajowan (Trachyspermum Ammi) Ethanolic Extract on A. Ochraceus Growth and Ochra- toxin Production," Turkish Journal of Biology, Vol. 33, No. 3, 2009, pp. 211-217.

[20] V. Polshettiwar and R. S. Varma "Microwave-Assisted Organic Synthesis and Transformations Using Benign Reaction Media," Accounts of Chemical Research, Vol. 41, No. 5, 2008, pp. 629-639. doi.org/10.1021/ar700238s

[21] S. S. Shankar, A. Rai, B. Ankamwar, A. Singh, A. Ahmad and M. Sastry, "Biological Synthesis of Triangular Gold Nanoprisms," Nature Materials, Vol. 3, June 2004, pp. 482-488. doi.org/10.1038/nmat1152

[22] K. Sau, A. L. Rogach, F. Jäckel, T. A. Klar and J. Feldmann. "Properties and Applications of Colloidal Nonspherical Noble Metal Nanoparticles," Advanced Materials, Vol. 22, No. 16, April 2010, pp. 1805-1825. doi.org/10.1002/adma.200902557

[23] M. A. Garcia, J. Venta, P. Crespo, J. Lopis, S. Penadés, A. Fernández and A. Hernando, "Surface Plasmon Resonance of Capped Au Nanoparticles," Physical Review B, Vol. 72, No. 24, December 2005, pp. 1-4. doi.org/10.1103/PhysRevB.72.241403

[24] R. A. Sperling and W. J. Parak, "Surface Modification, Functionalization and Bioconjugation of Colloidal Inorganic Nanoparticles," Philosophical Transactions of the Royal Society A, Vol. 368, No. 1915, March 2010, pp. 1333-1383.

[25] F. Chialva, F. Monguzzi, P. Manitto and A. Akgul. "Essential Oil Constituents of Trachyspermum Capticum (L.) Link Fruits," The Journal of Essential Oil Research, Vol. 5, No. 1, 1993, pp. 105-106.

[26] M. K. Juewon, A. Kanayama, K. Takahashi, et al., "In Vitro Free Radical Scavenging Activity of Platinum Nanoparticles," Nanotechnology, Vol. 20, No. 45, 2009, Article ID: 455105. doi.org/10.1088/0957-4484/20/45/455105 